\title{
REPRESENTATION THEOREMS FOR THE WEIERSTRASS TRANSFORM
}

\author{
Z. DITZIAN \\ (Received 10 November 1970) \\ Communicated by J. B. Miller
}

\section{Introduction}

In this paper we shall be interested in the Weierstrass transform defined by

$$
f(x)=\int_{-\infty}^{\infty} k(x-y, 1) d \alpha(y)
$$

converging (conditionally) for $x$ in some interval, where

$$
k(x, t)=(4 \pi t)^{-\frac{1}{2}} e^{-x^{2} / 4 t} .
$$

A representation theorem is a set of necessary and sufficient conditions on $f(x)$ so that $f(x)$ be represented by (1.1) with $\alpha(y)$ belonging to a certain class of functions. Representation theorems were discussed in [2], [3, Ch. VIII], [4], [5], [7] and [8]. In these papers conditions on $f(x)$ were given in order that $\alpha(y)$ would belong to one of the following classes:

(a) $\alpha(y)$ is increasing or decreasing, (see [8] and [3, p. 204]).

(b) $\alpha(y) \in B . V[-\infty, \infty]$, (see [7] and [3, p. 198]).

(c) $\alpha(y)$ satisfies $\int_{-\infty}^{\infty} k(x-y, 1)|d \alpha(y)|<\infty$ for all $x \in(a, b)$ for some $a, b a<b$, (see [4, p. 37] and [2]).

(d) $\alpha(y)=\int^{y} \phi(u) d u$ and $\phi \in L_{p}(-\infty, \infty) 1<p \leqq \infty$ (see [3, p. 195]).

(e) $\alpha(y)=\int^{y} \phi(u) d u$ and $e^{-(x-u)^{2} / 4} \phi(u) \in L_{p} 1<p \leqq \infty$ for $x \in(a, b)$ for some $a$ and $b$, (see [4, p. 43] and [2]).

(f) Same as (e) for $p=1$ (see [4, p. 48]).

(g) $|\phi(u)| \leqq N e^{a y^{2}}, a<\frac{1}{4}$ and $-\infty<y<\infty$, (see [3, p. 207]).

Obviously there are functions $f(x)$ representable by (1.1) with determining functions $\alpha(y)$. that are not in any one of the classes (a) $\rightarrow(\mathrm{g})$. Our main result will be to find necessary and sufficient conditions on $f(x)$ so that there exist a function $\alpha(y)$ locally of bounded variation for which (1.1) converges conditionally in some interval $(a, b) a<b$. This obviously is the widest class of $f(x)$ for which the Weierstrass-Stieltjes transform (1.1) exists. We may also restrict ourselves to the transform $f(x)$ 


$$
f(s)=\int_{-\infty}^{\infty} k(s-y, 1) \phi(y) d y,
$$

of locally Lebesgue integrable function $\phi(y)$. The widest class of $f(x)$ represented by (1.3) corresponds to the class of $\phi(y)$ for which (1.3) converges conditionally in a strip $a_{1}<\operatorname{Re} s<a_{2}$. Representation of this class is of special interest and will be the result of section 6 . New representation theorems will be given for $f(s)$ satisfying (1.1) and (1.3) where the integral converges absolutely in sections 5 and 7 respectively. A representation theorem for $f(s)$ satisfying (1.3) where

$$
|\phi(u)| \leqq \mathrm{Me}^{u^{2} / 4} \min \left(e^{-a u / 2}, e^{-b u / 2}\right)
$$

will be given in section 3. This result generalizes a corresponding result of Hirschman and Widder [3, p. 207], it is also used in proof and for motivation in the rest of the paper.

\section{A preliminary theorem for temperature functions}

To prove our representation theorem for Weierstrass transforms of functions satisfying (1.4) we first have to obtain a result about functions satisfying the Heat equation which is interesting by itself. To state this result we have to define class $H[3$, p. 181].

Definition 2.1. A function $u(x, t)$ is said to belong to class $H$ in domain $D$ if $u_{x x}(x, t)=u_{t}(x, t)$ and $u(x, t) \in C^{2}$ in $D$.

THEOREM 2.1. The conditions

$$
u(x, t) \in H \text { for } 0<t<1,-\infty<x<\infty
$$

and

$$
|u(x, t)| \leqq \frac{M}{\sqrt{1-t}} e^{2 / 4(1-t)} \min _{i=1,2} \exp \left[-\frac{a_{i} x}{2(1-t)}+\frac{a_{i}^{2} t}{4(1-t)}\right]
$$

for $0<t<1-\infty<x<\infty$ and some $a_{1}<a_{2}$, are necessary and sufficient that

$$
u(x, t)=\int_{-\infty}^{\infty} k(x-y, t) \phi(y) d y,
$$

where the integral (2.1) converges absolutely for $0<t<1,-\infty<x<\infty$ and $\phi(y)$ satisfies

$$
|\phi(y)| \leqq M e^{y^{2} / 4} \min _{i=1,2} e^{-a_{i} y / 2} \text { for all } y .
$$

To shorten some of the expressions we write 


$$
R\left(a_{i}, x, t\right)=\exp \left[-\frac{a_{i} x}{2(1-t)}+\frac{a_{i}^{2} t}{4(1-t)}\right] .
$$

Proof. We first prove the necessity of conditions (1) and (2). Condition (1) is implied by (2.1). Combining (2.1) and (2.2) we write:

$$
\begin{aligned}
|u(x, t)| \leqq & \int_{-\infty}^{\infty} k(x-y, t)|\phi(y)| d y \\
\leqq & M \int_{-\infty}^{\infty} k(x-y, t) e^{y^{2} / 4}\left\{\min _{i=1,2} e^{-a_{i} y / 2}\right\} d y \\
\leqq & M \min _{i=1,2} \int_{-\infty}^{\infty} k(x-y, t) \exp \left[\frac{1}{4} y^{2}-\frac{1}{2} a_{i} y\right] d y \\
= & M \min _{i=1,2} \frac{1}{\sqrt{4 \pi t}} \exp \left[\frac{x^{2}}{4(1-t)}-\frac{x a_{i}}{2(1-t)}+\frac{a_{i}^{2} t}{4(1-t)}\right] \\
& \cdot \int_{-\infty}^{\infty} \exp \left[-\frac{1-t}{4 t}\left(y-\frac{x}{1-t}+\frac{a_{i} t}{1-t}\right)^{2}\right] d y \\
\leqq & \frac{M}{\sqrt{1-t}} \exp \left(\frac{x^{2}}{4(1-t)}\right) \min _{i=1,2} \exp \left[-\frac{x a_{i}}{2(1-t)}+\frac{a_{i}^{2} t}{4(1-t)}\right]
\end{aligned}
$$

which completes the proof of necessity of condition (2).

We shall prove now the sufficiency of conditions (1) and (2). Define $V(x, t)$ by

$$
V(x, t)=\int_{-\infty}^{\infty} k(x-y, t) e^{y^{2} / 4}\left\{\min _{i=1,2} e^{-a_{i} y / 2}\right\} d y \equiv \int_{-\infty}^{\infty} k(x-y, t) d \beta(y)
$$

(Choosing $\beta(0)=0$ a normalized $\beta(y)$ is unique). Recalling $[1, \mathrm{p} .146(21)]$ that

$$
\int_{0}^{\infty} e^{-u^{2} / 4 \alpha} e^{-s u} d u=\pi^{\frac{1}{2}} \alpha^{\frac{1}{2}} e^{\alpha s^{2}} \operatorname{Erfc}\left(\alpha^{\frac{1}{2}} s\right)
$$

where $\operatorname{Erfc}(x)=2 \pi^{-\frac{1}{2}} \int_{x}^{\infty} e^{-t^{2}} d t$ we calculate $V(x, t)$ and obtain

$$
\begin{aligned}
V(x, t)=\frac{1}{2 \sqrt{1-t}} \exp \left(\frac{x^{2}}{4(1-t)}\right)\left\{R\left(a_{1}, x, t\right) \cdot \operatorname{Erfc}\left[\frac{x}{2\left(t-t^{2}\right)^{\frac{1}{2}}}-\frac{a_{1} t^{\frac{1}{2}}}{2(1-t)^{\frac{1}{2}}}\right]\right. \\
\left.+R\left(a_{2}, x, t\right) \operatorname{Erfc}\left[-\frac{x}{2\left(t-t^{2}\right)^{\frac{1}{2}}}+\frac{a_{2} t^{\frac{1}{2}}}{2(1-t)^{\frac{3}{2}}}\right]\right\} .
\end{aligned}
$$

Obviously the necessity of (1) and (2) implies

$$
V(x, t) \leqq \frac{1}{\sqrt{1-t}} \exp \left(\frac{x^{2}}{4(1-t)}\right) \min _{i=1,2} R\left(a_{i}, x, t\right) \equiv H(x, t) .
$$

We shall need in our proof that for every fixed $a_{1}$ and $a_{2}$ and $\varepsilon>0$ $0<t<\delta(\varepsilon)$ 


$$
V(x, t) \geqq(1-\varepsilon) H(x, t) .
$$

We can choose $\eta_{1}(\varepsilon)$ so that for $|y| \leqq \eta_{1}(\varepsilon) e^{y^{2} / 4} \min _{i=1,2} e^{-a_{i} y / 2} \geqq 1-\varepsilon / 3$ and then using (2.3) we obtain for $|x| \leqq \frac{1}{2} \eta_{1}(\varepsilon)$ and $0<t<\delta_{1}(\varepsilon) V(x, t) \geqq 1-2 \varepsilon / 3$. Since $H(x, t)$ is continuous at a neighbourhood of $(0,0)$ and $H(0,0)=1$ we have for $|x| \leqq \eta_{2}$ and $0<t<\delta_{2} V(x, t) \geqq(1-\varepsilon) H(x, t)$. For $|x| \geqq \eta_{2}$ we can choose $\delta_{3}<\delta_{2}$ such that for $t<\delta_{3}$

$$
\min _{i=1,2} R\left(a_{i}, x, t\right)= \begin{cases}R\left(a_{1}, x, t\right) & x \leqq-\eta_{2} \\ R\left(a_{2}, x, t\right) & x \geqq \eta_{2} .\end{cases}
$$

To prove (2.7) it is enough now to show for $|t|<\delta \leqq \delta_{3}$ and $x \geqq \eta_{2}$ that

$$
\begin{aligned}
\operatorname{Erfc}\left[\frac{x}{2\left(t-t^{2}\right)^{\frac{1}{2}}}-\frac{a_{1} t^{\frac{1}{2}}}{2(1-t)^{\frac{1}{2}}}\right] & <\frac{\varepsilon}{2} \exp \left[\frac{\left(a_{2}^{2}-a_{1}^{2}\right) t}{4(1-t)}+\frac{\left(a_{1}-a_{2}\right) x}{2(1-t)}\right] \\
& <\frac{\varepsilon}{2} \exp \left[\frac{\left(a_{2}^{2}-a_{1}^{2}\right) t}{4(1-t)}\right],
\end{aligned}
$$

$$
\operatorname{Erfc}\left[-\frac{x}{2\left(t-t^{2}\right)^{\frac{1}{2}}}+\frac{a_{2} t^{\frac{1}{2}}}{2(1-t)^{\frac{1}{2}}}\right]>2-\varepsilon
$$

and corresponding results for $x \leqq-\eta_{2}$. Using the estimate

$$
\int_{x}^{\infty} e^{-y^{2}} d y \leqq \int_{x}^{\infty} y e^{-y^{2}} d y=\frac{1}{2} e^{-x^{2}}
$$

for $x \geqq 1$ and straightforward computation we can prove (a) and (b) and therefore (2.7).

We recall now that (see Th. 12.2 of $[3$, p. 202]) necessary and sufficient conditions for $u(x, t)$ to be written as

$$
u(x, t)=\int_{-\infty}^{\infty} k(x-y, t) d \alpha(y) \text { in } 0<t<\delta,-\infty<x<\infty
$$

with $\alpha(y)$ nondecreasing is

$$
u(x, t) \geqq 0 \text { and } u(x, t) \in H \quad \text { for } 0<t<\delta,-\infty<x<\infty .
$$

Using (2.7) we have

$$
-M(1-\varepsilon)^{-1} V(x, t) \leqq u(x, t) \leqq M(1-\varepsilon)^{-1} V(x, t) \quad 0<t<\delta(\varepsilon),
$$

and this implies the existence of $\gamma_{i}(t) i=1,2$, both nondecreasing and unique after normalization, such that

(2.8) $M(1-\varepsilon)^{-1} V(x, t)+(-1)^{i} u(x, t)=\int_{-\infty}^{\infty} k(x-y, t) d \gamma_{i}(y) \quad 0<t<\delta i=1,2$. 
Recalling (2.4) there exists $\alpha(y)$ locally of bounded variation such that

$$
u(x, t)=\int_{-\infty}^{\infty} k(x-y, t) d \alpha(y)
$$

for $0<t<\delta(\varepsilon)$ and $\gamma_{i}(y)=M(1-\varepsilon)^{-1} \beta(y)+(-1)^{i} \alpha(y)$. Following now arguments in [7] and [3, p. 207] we get $\alpha(y)=\int^{y} \phi(x) d x$ and

$$
|\phi(y)| \leqq M(1-\varepsilon)^{-1} e^{y^{2} / 4} \min _{i=1,2} e^{-a_{i} y / 2} .
$$

The function $\alpha(y)$ is independent of $\varepsilon$, in spite of the dependence of $\gamma_{i}(y)$ on $\varepsilon$, since $\alpha(y)$ satisfying (2.9) in $0<t<\delta$ is unique. $\phi(y)$ now satisfies $(2.10)$ for all $\varepsilon$ and therefore (2.2) but then (2.1) converges absolutely in $0<t<1$.

REMARK 2.1.a. In condition (2) of Theorem 2.1 we replace $0<t<1$ by $0<t<\delta$ and call it (2)*. Conditions (1) of Theorem 2.1 and (2)* can replace (1) and (2) as necessary and sufficient for (2.1) and (2.2). The necessity is obvious while sufficiency follows the proof of Theorem 2.1.

\section{The asymptotic representation theorem}

In this section a representation theorem for the Weierstrass transform of $\phi$ satisfying (1.3) will be obtained. This result will be used in the motivation and proof of the following theorems of this paper. For our theorem we define first, class $A[a, b]$.

Definition 3.1. A function $f(z)$ analytic in $a<R e z<b$ belongs to class $A[a, b]$ if $f(x+i y)=0\left(e^{y^{2} / 4}\right)$ uniformly for $x$ in every closed subinterval of $(a, b)$.

Define also (see [3]) $K(s, t)$ by

$$
K(s, t)=\left(\frac{\pi}{t}\right)^{\frac{1}{2}} e^{s^{2} / 4 t}=2 \pi k(i s, t) .
$$

THEOREM 3.1. The conditions (1) $f(z) \in A\left[a_{1}, a_{2}\right]$ and

$$
\left|\frac{1}{2 \pi} \int_{d-i \infty}^{d+i \infty} K(s-x, t) f(s) d s\right| \leqq M t^{-\frac{1}{2}} e^{x^{2} / 4 t} \min _{i=1,2} R\left(a_{i}, x, 1-t\right)
$$

(where $R\left(a_{i}, x, t\right)$ was defined by (2.3)) for some $d, a_{1}<d<a_{2}$ and $0<r<1$ are necessary and sufficient that

$$
f(x)=\int_{-\infty}^{\infty} k(x-y, 1) \phi(y) d y
$$

converges absolutely for $a_{1}<x<a_{2}$ and

$$
|\phi(y)| \leqq M e^{y^{2} / 4} \min _{i=1,2} e^{-a_{i} y / 2} .
$$


Proof. To prove necessity of (1) and (2) we observe that (3.3) implies $\mid \exp \left[-(x-y)^{2} / 4\right] \phi(y) \|_{1}<\infty$ for $a_{1}<x<a_{2}$ and therefore using Lemma 1 of [4, p. 32] (3.3) implies condition (1). Using Theorem 7.3 of [3, pp. 189-191] we obtain for $a_{1}<d<a_{2}$

$$
\frac{1}{2 \pi i} \int_{d-i \infty}^{d+i \infty} K(z-x, t) f(z) d z=\int_{-\infty}^{\infty} k(x-u, 1-t) \phi(u) d u .
$$

The necessity of condition (2) follows now the corresponding part of Theorem 2.1 replacing $t$ by $1-t$.

To prove (1) and (2) are sufficient we define

$$
u(x, 1-t)=\frac{1}{2 \pi i} \int_{d-i \infty}^{d+i \infty} K(s-x, t) f(s) d s .
$$

Using Cauchy's theorem and the asymptotic behaviour of both $K(s, t)$ and $f(s)$ it follows that (3.5) is independent of $d$, provided $d$ satisfies $a_{1}<d<a_{2}$. Recalling that $(\partial / \partial x)^{2} K(s-x, t)=-(\partial / \partial t) K(s-x, t)$ and differentiating under the integral sign in (3.5), which is easily justified, we obtain

$$
\left(\frac{\partial}{\partial x}\right)^{2} u(x, 1-t)=-\frac{\partial}{\partial t} u(x, 1-t) \text { for } 0<t<1 \text { and }-\infty<x<\infty .
$$

The sufficiency part of Theorem 2.1 implies now

$$
u(x, 1-t)=\int_{-\infty}^{\infty} k(x-y, 1-t) \phi(y) d y \text { for } 0<t<1-\infty<x<\infty
$$

where $\phi(y)$ satisfies (3.3). For such $\phi(y)$

$$
f_{*}(x) \equiv \int_{-\infty}^{\infty} k(x-y, 1) \phi(y) d y
$$

converges absolutely for $a_{1}<x<a_{2}$. To complete the proof it will be sufficient to show $f_{*}(x)=f(x)$ on $a_{1}<x<a_{2}$. Using the Lebesgue convergence theorem we obtain

$$
f_{*}(x)=\lim _{t \rightarrow 0+} u(x, 1-t)=\lim _{t \rightarrow 0+} \int_{-\infty}^{\infty} k(x-y, 1-t) \phi(y) d y .
$$

Combining (3.5) and (3.9) we have

$$
\begin{aligned}
f_{*}(x) & =\lim _{t \rightarrow 0+} \frac{1}{2 \pi i} \int_{d-i \infty}^{d+i \infty} K(s-x, t) f(s) d s \\
& =\lim _{t \rightarrow 0+} \frac{1}{2 \pi i} \int_{x-i \infty}^{x+i \infty} K(s-x, t) f(s) d s \\
& =\lim _{t \rightarrow 0+} \frac{1}{2 \pi} \int_{-\infty}^{\infty} K(i y, t) f(x+i y) d y=\lim _{t \rightarrow 0+} \int_{-\infty}^{\infty} k(y, 1) f(x+i y \sqrt{t} d y .
\end{aligned}
$$


Since for $x \in\left[A_{1}, A_{2}\right], \quad a_{1}<A_{1}<A_{2}<a_{2}$ and $0<t \leqq 1-\delta \quad \int_{-\infty}^{\infty} k(y, 1)$ $|f(x+i y \sqrt{t})| d y \leqq M \int_{-\infty}^{\infty} e^{-y^{2} / 4} e^{y^{2}(1-\rho) / 4} d y<\infty$ we can use again Lebesgue convergence theorem now to show that $f(x)=\lim _{t \rightarrow 0+} \int_{-\infty}^{\infty} k(y, 1) f(x+i y \sqrt{t}) d y$ which completes the proof.

We conclude this section with a few remarks. We shall define first, class $B(a, b)$.

Definition 3.2. A function $f(z)$ analytic in $a<\operatorname{Re} z<b$ belongs to class $B[a, b]$ if $f(x+i y)=0\left(y e^{y^{2} / 4}\right)|y| \rightarrow \infty$ uniformly for every closed subinterval of $(a, b)$.

REMARK 3.1.a. In a related result of Hirschman and Widder [3, p. 207] where the Weierstrass transform of $\phi(y)$ satisfying $|\phi(y)| \leqq M e^{a y^{2}} 0<a<\frac{1}{4}$ is represented the condition $f(z) \in B[a, b]$ is required. Using Nessel's result $[4, \mathbf{p}$. $31]$ in the theorem above $[3$, p. 207] we can assume there $f(z) \in A[a, b]$ instead of $f(z) \in B[a, b]$.

REMARK 3.1.b. If we follow carefully the sufficiency proof of Theorem 3.1 we can see that $f(z) \in B[a, b]$ can replace $f(z) \in A[a, b]$ there. (The necessity parts is easier then).

REMARK 3.1.c. In fact, in both theorems $f(z)=0\left(|y|^{n} e^{y^{2} / 4}\right) y \rightarrow \infty$ uniformly in any closed subinterval of $(a, b)$ can replace $A[a, b]$ and $B[a, b]$. But we do use only $B[a, b]$ for theorems that will be proved later in this paper.

REMARK 3.1.d. In theorem 3.1 in condition (2) $0<t<1$ could be replaced by $1-\delta<t<1$. This follows from Remark 2.1.a since Theorem 3.1 uses for its sufficiency part, the sufficiency part of Theorem 2.1 with $1-t$ replacing $t$.

\section{Functions of locally bounded variation whose Weierstrass transform converges conditionally}

In this section the most general class of functions $\alpha(y)$ for which WeierstrassStieltjes transform is defined will be treated.

THEOREM 4.1. The conditions

(1) $f(z) \in B\left[a_{1}, a_{2}\right] a_{1}<a_{2}$ (Def. 3.2).

(2) For some $d a_{1}<d<a_{2}$

$$
\left|\int_{0}^{x}\left\{\int_{d-i \infty}^{d+i \infty} K(s-\xi, t) f(s) d s\right\} d \xi\right| \leqq M\left(\alpha_{1}, \alpha_{2}\right) t^{-\frac{1}{2}} e^{x^{2} / 4 t} \min _{i=1,2} R\left(\alpha_{i}, x, 1-t\right)
$$

for all $\alpha_{i}$ satisfying $a_{1}<\alpha_{1}<\alpha_{2}<a_{2}, 0<t<1$ and $-\infty<x<\infty$; and 


$$
\int_{a}^{b}\left|\int_{d-i \infty}^{d+i \infty} K(s-\xi, t) f(s) d s\right| d \xi \leqq L(a, b) \quad 1-\delta \leqq t<1
$$

for any $(a, b)-\infty<a<b<\infty$; are necessary and sufficient that; $f(x)$ will be represented as $f(x)=\int_{-\infty}^{\infty} k(x-y, 1) d \alpha(t)$ where the integral converges conditionally for $a_{1}<x<a_{2}$.

REMARK 4.1.a. Actually we shall prove that (1) (2) for a fixed pair $\left(\alpha_{1} \alpha_{2}\right)$ and (3) will imply the conditional convergence of (1.1) for $x \in\left(\beta_{1}, \beta_{2}\right) \alpha_{1}<\beta_{1}<\beta_{2}<\alpha_{2}$ and that will imply (1), (2) and (3) with $\gamma_{i}$ instead of $\alpha_{i}$ where $\beta_{1}<\gamma_{1}<\gamma_{2}<\beta_{2}$.

Proof. We shall show (1), (2) and (3) are necessary first. The necessity of (1) follows $\left[3\right.$, p. 180]. The conditional convergence of $(1.1)$ in $\left(a_{1}, a_{2}\right)$ implies (see [3, p. 190]).

$$
|\alpha(y)| \leqq M\left(\alpha_{1}, \alpha_{2}\right) e^{y^{2} / 4} \min _{i=1,2} e^{-\alpha_{i} y / 2}
$$

for any $\left(\alpha_{1}, \alpha_{2}\right)$ satisfying $a_{1}<\alpha_{1}<\alpha_{2}<a_{2}$, and also for $a_{1}<d<a_{2}$

$$
\frac{1}{2 \pi i} \int_{d+i \infty}^{d+i \infty} K(s-x, t) f(s) d s=\int_{-\infty}^{\infty} k(x-u, 1-t) d \alpha(u) .
$$

Writing now

$$
\begin{aligned}
\int_{0}^{x}\left\{\int_{-\infty}^{\infty} k(\xi-u, 1-t) d \alpha(u)\right\} d \xi= & \int_{0}^{x}\left\{\int_{-\infty}^{\infty}\left[\frac{\partial}{\partial u} k(\xi-u, 1-t)\right] \alpha(u) d u\right\} d \xi \\
= & -\int_{-\infty}^{\infty} \int_{0}^{x} \frac{\partial}{\partial \xi} k(\xi-u, 1-t) \alpha(u) d \xi d u \\
= & -\int_{-\infty}^{\infty} k(x-u, 1-t) \alpha(u) d u \\
& +\int_{-\infty}^{\infty} k(-u, 1-t) \alpha(u) d u \equiv I_{1}+I_{2} .
\end{aligned}
$$

The interchange of order of integration above is justified by Fubini theorem using (4.1). Theorem 3.1 used on both $I_{1}$ and $I_{2}$ implies condition (2). Recalling that $\alpha(y)$ satisfies $\int_{a-1}^{b+1}|d \alpha(y)| \leqq A_{*}(a, b)(\alpha(y)$ is locally of bounded variation), we have

$$
\begin{aligned}
\int_{a}^{b}\left|\int_{-\infty}^{\infty} K(\xi-u, 1-t) d x(u)\right| d \xi \leqq & 2 \pi\left\{\int_{a}^{b}\left|\int_{-\infty}^{a-1} k(\xi-u, 1-t) d x(u)\right| d \xi\right. \\
& +\int_{a}^{b}\left|\int_{a-1}^{b+1} k(\xi-u, 1-t) d x(u)\right| d \xi \\
& \left.+\int_{a}^{b}\left|\int_{b+1}^{\infty} k(\xi-u, 1-t) d \alpha(u)\right| d \xi\right\} \\
& \equiv 2 \pi\left\{J_{1}+J_{2}+J_{3}\right\} .
\end{aligned}
$$


It is easy to see that $J_{2} \leqq A_{*}(a, b)$. To estimate $J_{1}$ (treatment of $J_{3}$ is similar) we write

$$
\begin{array}{r}
J_{1} \leqq \int_{a}^{b}\left|\int_{-\infty}^{a-1} \frac{\partial}{\partial \xi} k(\xi-u, 1-t) x(u) d u\right| d \xi+\int_{a}^{b}|\alpha(a-1)| k(\xi-u, 1-t) d \xi \\
=J_{1,1}^{*}+J_{1,2}^{*} .
\end{array}
$$

Obviously $J_{1,2}^{*}$ is bounded independently of $t$ (we choose $\alpha(0)=0$ ). For $\xi \in(a, b)$ and $u \in(-\infty, a-1) \quad \partial / \partial \xi k(\xi-u, 1-t)>0$

$$
\begin{aligned}
J_{1,1}^{*} & \leqq \int_{a}^{b}\left\{\int_{-\infty}^{a-1} \frac{\partial}{\partial \xi} k(\xi-u, 1-t)|\alpha(u)| d u\right\} d \xi \\
& \leqq \int_{-\infty}^{a-1} k(a-u, 1-t)|\alpha(u)| d u+\int_{-\infty}^{a-1} k(b-u, 1-t)|\alpha(u)| d u \\
& =0(1) t \rightarrow 1-
\end{aligned}
$$

Therefore $J_{2}$ is bounded for $1-\delta<t<1$ which completes the proof of condition (3).

To prove that conditions (1), (2) and (3) are sufficient our first step will be to show for a fixed $t \quad 0<t<1$ and for $x \in\left(a_{1}, a_{2}\right)$

$$
\int_{-\infty}^{\infty} k(x-\xi, t)\left\{\frac{1}{2 \pi i} \int_{d-i \infty}^{d+i \infty} K(s-\xi, t) f(s) d s\right\} d \xi=f(x) .
$$

Condition (2) implies the convergence of the integral in (4.3) (conditional convergence). Condition (1) combined with Cauchy Theorem implies for $a_{1}<d_{1}$, $d<a_{2}$

$$
\int_{d-i \infty}^{d+i \infty} K(s-\xi, t) f(s) d s=\int_{d_{1}-i \infty}^{d_{1}+i \infty} K(s-\xi, t) f(s) d s .
$$

Straightforward computation yields for $0<\tau<t<1$

$$
\int_{-\infty}^{\infty} k(x-\xi, \tau)\left\{\int_{d-i \infty}^{d+i \infty}|K(s-\xi, t) f(s)| d s\right\} d \xi<\infty .
$$

Therefore using (4.4) and (4.5) for $a_{1}<x<a_{2}$ and [3, p. 177, (1)] we have

$$
\begin{aligned}
\int_{-\infty}^{\infty} k(x-\xi, \tau) & \left.\frac{1}{2 \pi i} \int_{d-i \infty}^{d+i \infty} K(s-\xi, t) f(s) d s\right\} d \xi \\
& =\frac{1}{2 \pi i} \int_{x-i \infty}^{x+i \infty} f(s)\left\{\int_{-\infty}^{\infty} k(x-\xi, \tau) K(s-\xi, t) d \xi\right\} d s \\
& =\int_{-\infty}^{\infty} f(x+i y) d y \int_{-\infty}^{\infty} k(\xi, \tau) k(i \xi-u, t) d \xi \\
& =\int_{-\infty}^{\infty} f(x+i y) k(y, t-\tau) d y
\end{aligned}
$$


Obviously

$$
\lim _{\tau \rightarrow t^{-}} \int_{-\infty}^{\infty} f(x+i y) k(y, t-\tau) d y=f(x)
$$

and therefore to prove (4.3) it is enough to show

$$
\left|\int_{-\infty}^{\infty}(k(x-\xi, \tau)-k(x-\xi, t))\left\{-\frac{1}{2 \pi i} \int_{d-i \infty}^{d+i \infty} K(s-\xi, t) f(s) d s\right\} d \xi\right|=o(1) \quad \tau \rightarrow t-
$$

which we can obtain applying condition (2) again.

Our next step will be to determine $\alpha(y)$. We define $\alpha_{t}(y)$ by

$$
\alpha^{t}(y)=\int_{0}^{y}\left\{\int_{d-i \infty}^{d+i \infty} K(s-\xi, t) f(s) d s\right\} d \xi .
$$

Using condition (3) and Helly-Bray's Theorem $[5, \mathrm{p} .31]$ there exist a sequence $t_{n}$ and a function $\alpha(y), y \in[a, b]$ such that $\lim _{n \rightarrow \infty} \int_{a}^{b} f(y) d \alpha_{t_{n}}(y)=\int_{a}^{b} g(y) d \alpha(y)$ for all $g(y) \in[a, b]$ where $\int_{a}^{b}|d \alpha(y)| \leqq L(a, b)$ and $\alpha_{t_{n}}(y)$ tend to $\alpha(y)$ at all points of continuity of $\alpha(y)$. We take the sequence $\alpha_{t_{n(t)}}(y)$ to correspond to $[-1,1]$ (for $[a . b])$ and a subsequence of $\alpha_{t_{(n 1)}} y, \alpha_{t_{(n 2)}}(y)$ to correspond to $[-2,2]$ etc. Define now $\alpha_{t(m)}(y)$ by Cantor diagonal selection principle. It seems as if we have different functions $\alpha(n, y)$ for each interval $[-n, n]$ but normalizing the $\alpha(n, y)$ and recalling that $\alpha_{t_{n(k)}}(y)$ is a subsequence of $x_{t_{n(k-1)}}(y)$ we observe that a unique function $\alpha(y)$ exists, is locally of bounded variation, satisfies

$$
\lim _{m \rightarrow \infty} \int_{-n}^{n} g(y) d \alpha_{t(m)}(y)=\int_{-n}^{n} g(y) d \alpha(y) \forall n
$$

and $\lim _{m \rightarrow \infty} \alpha_{t(m)}(y)=\alpha(y)$ at all points of continuity of $\alpha(y)$ (that is at all but a countable set of points). Therefore, for any $\left(\alpha_{1} \alpha_{2}\right) a_{1}<\alpha_{1}<\alpha_{2}<a_{2}$

$$
\begin{aligned}
|\alpha(y)| \leqq & \lim _{t(n) \rightarrow 1-}\left|\alpha_{t(n)}(y)\right| \leqq M\left(\alpha_{1} \alpha_{2}\right) \lim _{t(n) \rightarrow 1-}|t(n)|^{-\frac{1}{2}} e^{y^{2} / 4 t(n)} \\
& \cdot \min _{i=1,2} R\left(\alpha_{i}, y, 1-t(n)\right) \leqq M\left(\alpha_{1}, \alpha_{2}\right) e^{y^{2} / 4} \min _{i=1,2} e^{-\alpha_{i} y / 2}
\end{aligned}
$$

This implies

$$
f_{*}(x) \equiv \int_{-\infty}^{\infty} k(x-y, 1) d \alpha(y)
$$

converges conditionally in $a_{1}<x<a_{2}$. The above means that for $A \leqq A_{1}$ and $B \geqq B_{1}$ for a fixed $x, x \in\left(a_{1}, a_{2}\right)$

$$
\left|\int_{A}^{B} k(x-y, 1) d \alpha(y)-f_{*}(x)\right|<\varepsilon .
$$

Using condition (2) one can show recalling (4.3) that for $A \leqq A_{2}<A_{1}$ and $B \geqq B_{2}>B_{1}$ and $t, t_{0} \leqq t<1$ 


$$
\left|\int_{A}^{B} k(x-y, t) d \alpha_{t}(y)-f(x)\right|<\varepsilon
$$

(where $A_{2}$ and $B_{2}$ are independent of $t$ ). Choose $A=-N B=N$. For $t$ satisfying $t_{0}<t_{*}<t<1$ we have

$$
\left|\int_{-N}^{N}(k(x-y, 1)-k(x-y, t)) d \alpha_{t}(y)\right|<\varepsilon .
$$

Choosing $t(m)>t\left(m_{0}\right)>t_{*}$ we have

$$
\left|\int_{-N}^{N} k(x-y, 1) d \alpha_{t(m)}(y)-\int_{-N}^{N} k(x-y, 1) d \alpha(y)\right|<\varepsilon .
$$

Combining (4.9), (4.10), (4.11) and (4.12) we have $\left|f(x)-f_{*}(x)\right|<4 \varepsilon$. But both $f(x)$ and $f_{*}(x)$ are independent of $N$ and $t$ and therefore $f(x)=f_{*}(x)$. The above being true for $a_{1}<x<a_{2}$ we have

$$
f(x)=\int_{-\infty}^{\infty} k(x-y, 1) d \alpha(y) \quad a_{1}<x<a_{2}
$$

which completes the proof of our theorem.

\section{Absolute convergence}

In this section necessary and sufficient conditions on $f(x)$ to be represented as absolutely convergent Weierstrass-Stieltjes transform will be achieved. We shall need the following definition:

Definition 5.1. A function $f(z)$ analytic in the strip $a<\operatorname{Re} z<b$ and satisfying $f(x+i y)=0\left(e^{y^{2} / 4}\right)$ uniformly in any closed subinterval belongs to class $C[a, b]$.

THEOREM 5.1. The conditions

$$
f(x) \in C\left[a_{1}, a_{2}\right], a_{1}<a_{2}
$$

and

$$
\int_{0}^{x}\left|\int_{d-i \infty}^{d+i \infty} K(s-\xi, t) f(s) d s\right| d \xi \leqq M\left(\alpha_{1}, \alpha_{2}\right) t^{-\frac{1}{2}} e^{x^{2} / 4 t} \min _{i=1,2} R\left(\alpha_{i}, x, 1-t\right)
$$

where $a_{1}<d<a_{2}, 0<t<1,-\infty<x<\infty$ and $\alpha_{1}, \alpha_{2}$ are any pair satisfying $a_{1}<\alpha_{1}<\alpha_{2}<a_{2} ;$ are necessary and sufficient that; $f(z)=\int_{-\infty}^{\infty} k(z-y, 1) d \alpha(y)$ and the integral will converge absolutely for $a_{1}<\operatorname{Re} z<a_{2}$.

Proof. The necessity proof of (2) is computational and that of (1) follows $[4$, p. 32]. 
To prove the sufficiency of (1) and (2) we observe that these conditions imply conditions (1), (2) and (3) of Theorem 4.1 and therefore the conditional convergence of $f(x)=\int_{-\infty}^{\infty} k(x-y, 1) d \alpha(y)$. We can complete the proof if we show that $f_{*}(x)=\int_{-\infty}^{\infty} k(x-y, 1)|d \alpha(y)|<\infty$ for $a_{1}<x<a_{2}$. We recall that

$$
\int_{0}^{u}|d \alpha(y)| \leqq \lim _{n \rightarrow \infty} \int_{0}^{u}\left|d \alpha_{t_{n}}(y)\right|
$$

where $\alpha_{t}(y)$ was defined in (4.7). Condition (2) implies now for $\alpha_{1}<x<\alpha_{2}$

$$
\begin{aligned}
\int_{0}^{u}|d \alpha(y)| & \leqq M \lim _{t \rightarrow 1-} e^{u^{2} / 4 t} \min R\left(\alpha_{i}, u, 1-t\right) \\
& \leqq M e^{u^{2} / 4} \min _{i=1,2} e^{-\alpha_{i} u / 2} .
\end{aligned}
$$

The last estimate establishes the absolute convergence of $\int_{-\infty}^{\infty} k(x-y, 1) d \alpha(y)$.

REMARK 5.1.a. One can observe that the class of functions $\alpha(t)$ is the same as treated by Nessel [4, p. 37]; the conditions are different however. Condition (2) here replaces Nessel's condition

$\left\|\exp \left[-(t-x)^{2} / 4\right] \cdot \frac{1}{\sqrt{4 \pi i}} \int_{x-i T}^{x+i T}\left(1-\frac{|y|}{T}\right) \exp \left[(s-x)^{2} / 4\right] f(s) d s\right\|_{L_{1}}=o(1)$

for all $T$. Also here most of the proof follows as a corollary of the representation of the more general class.

\section{Weierstrass transform of locally Lebesgue integrable functions}

Representation theorem for Weierstrass transform

$$
f(x)=\int_{-\infty}^{\infty} k(x-y, 1) \phi(y) d y
$$

where $\phi(y)$ is locally Lebesgue integrable and (6.1) converges conditionally in some strip would be obtained as follows:

THEOREM 6.1. Conditions (1) and (2) of Theorem 4.1 and

$$
\text { (3)* } \int_{a}^{b}\left|\int_{d-i \infty}^{d+i \infty}\left[K\left(s-\xi, t_{1}\right)-K\left(s-\xi, t_{2}\right)\right] f(s) d s\right| d \xi=o(1) t_{i} \rightarrow 1-, 1-\delta<t_{\imath}<1
$$

for any $a, b-\infty<a<b<\infty$ (but the rate at which the double integral tends to zero depends on $(a, b))$, are necessary and sufficient for $f(x)$ to be represented by (6.1) converging conditionally in $a_{1}<x<a_{2}$ and $\phi(y) \in L_{1}(a, b)$ for all $-\infty<a<b<\infty$. 
Proof. Condition (1) and (2) are necessary since they were necessary already for Theorem 4.1. To prove (3) we write

$$
\begin{aligned}
& \int_{a}^{b}\left|\frac{1}{2 \pi i} \int_{d-i \infty}^{d+i \infty}\left[K\left(s-\xi, t_{1}\right)-K\left(s-\xi, t_{2}\right)\right] f(s) d s\right| d \xi \\
& \quad=\int_{a}^{b}\left|\int_{-\infty}^{\infty}\left[k\left(\xi-y, 1-t_{1}\right)-k\left(\xi-y, 1-t_{2}\right)\right] \phi(y) d y\right| d \xi \\
& \leqq \sum_{i=1}^{2} \int_{a}^{b}\left|\int_{-\infty}^{\infty} k\left(\xi-y, 1-t_{i}\right) \phi(y) d y-\phi(\xi)\right| d \xi \equiv \sum_{i=1}^{2} I_{i} .
\end{aligned}
$$

To estimate $I_{1}\left(I_{2}\right.$ is estimated similarly) we follow the proof of Theorem 4.1 and write

$$
I_{1}=\int_{a}^{b}\left|\int_{a-1}^{b+1} k\left(\xi-y, 1-t_{1}\right) \phi(y) d y-\phi(\xi)\right| d \xi+o(1) \quad t \rightarrow 1-.
$$

For a fixed $\varepsilon$ there exist $N$ such that $\int_{-N}^{N} k(x, 1) d x \geqq 1-\varepsilon$ and therefore for $\left(1-t_{1}\right) N<1$ we write

$$
\begin{aligned}
I_{1}=\int_{a}^{b} & \left|\int_{-N}^{N} k(v, 1)\left[\phi\left(\xi+\sqrt{1-t_{1} v}\right)-\phi(\xi)\right] d v\right| d \xi+\varepsilon \int_{a}^{b}|\phi(\xi)| d \xi \\
& +\varepsilon \int_{a-1}^{b+1}|\phi(y)| d y+0(1) \quad t \rightarrow 1-.
\end{aligned}
$$

We now use Fubini's Theorem to write

$$
I_{1} \leqq \int_{-N}^{N} k(v, 1)\left\{\int_{a}^{b}\left|\phi\left(\xi+\sqrt{1-t_{1} v}\right)-\phi(\xi)\right| d \xi\right\} d v+2 \varepsilon \int_{a-1}^{b+1}|\phi(\xi)| d \xi .
$$

Recalling that $\tau(h)=\int_{a}^{b}|\phi(\xi+h)-\phi(\xi)| d \xi$ satisfies $\tau(h)=o(1) h \rightarrow 0+$ we complete the proof of condition (3).

To prove sufficiency we recall that conditions (1) (2) and (3)* imply the corresponding conditions of Theorem 4.1 and therefore

$$
f(x)=\int_{-\infty}^{\infty} k(x-y, 1) d \alpha(y) .
$$

Condition (3)* implies $\alpha(y)=\int^{y} \phi(u) d u$ and this completes the proof of our theorem.

\section{Absolutely convergent Weierstrass transform}

The following theorem corresponding to those of former section can be obtained.

THEOREM 7.1. The condition (1) $f(z) \in A\left[a_{1}, a_{2}\right],(2)$ condition (2) of Theorem 5.1 , and (3) condition (3)* of Theorem 6.1, are necessary and sufficient for $f(x)$ 
to be written as $f(x)=\int_{-\infty}^{\infty} k(x-y, 1) \phi(y) d y$ the integral converging absolutely for $a_{1}<x<a_{2}$.

The proof is similar to proof of former theorems in this paper and would not be given here.

The same class of functions has also a different representation theorem $[4$, p. 48 , Satz 3].

\section{References}

[1] Erdélyi (and others), Tables of integral transforms, Vol. I (McGraw Hill, 1954).

[2] H. P. Heinig, 'Representation of functions as Weierstrass-transforms', Canadian Mathematical Bulletin 10 (1967), 711-722.

[3] I. I. Hirschman and D. V. Widder, The Convolution Transform (Princeton Univ. Press, 1955).

[4] R. J. Nessel, 'Ueber die Darstellung holomorpher Funktionen durch Weierstrass and Weierstrass-Stieltjes Integrale', Journal fur die reine und angewandte Mathematik (1965), 31-50.

[5] H. Pollard, 'Representation as Gaussian integral', Duke Math. Jour. 10 (1943), 59-65.

[6] D. V. Widder, The Laplace transform. (Princeton Univ. Press, 1946).

[7] D. V. Widder, 'Necessary and sufficient conditions for representation of a function by a Weierstrass transform', Trans. Amer. Math. Soc. 71 (1951), 430-439.

[8] D. V. Widder, 'Weierstrass transforms positive functions', Proc, of Nat. Acad. of Science 37 (1951), 315-317.

Department of Mathematics

University of Alberta

Canada 